\title{
PROGRAMMING PEARL \\ Enhancing a Search Algorithm to Perform Intelligent Backtracking
}

\author{
MAURICE BRUYNOOGHE \\ Katholieke Universiteit Leuven, Department of Computer Science \\ Celestijnenlaan 200A, B3001 Heverlee, Belgium \\ e-mail: Maurice.Bruynooghe@cs.kuleuven.ac.be
}

\begin{abstract}
This paper illustrates how a Prolog program, using chronological backtracking to find a solution in some search space, can be enhanced to perform intelligent backtracking. The enhancement crucially relies on the impurity of Prolog that allows a program to store information when a dead end is reached. To illustrate the technique, a simple search program is enhanced.
\end{abstract}

KEYWORDS: intelligent backtracking, dependency-directed backtracking, backjumping, conflict-directed backjumping, nogood sets, look-back.

\section{Introduction}

The performance of backtracking algorithms for solving finite-domain constraint satisfaction problems can be improved substantially by so called look-back and look-ahead methods (Dechter and Frost 2002). Look-back techniques extract information by analyzing failing search paths that are terminated by dead ends and use that information to prune the search tree. Look-ahead techniques use constraint propagation algorithms in an attempt to avoid such dead ends altogether. Constraint propagation can rather easily be isolated from the search itself and can be localized in a constraint store. Following the seminal work of (Van Hentenryck 1989), look-ahead techniques are available to the logic programmer in a large number of systems.

This is not the case for look-back methods. Intelligent backtracking has been explored as a way of improving the backtracking behavior of logic programs (Bruynooghe and Pereira 1984). For some time, a lot of effort went into adding intelligent backtracking to Prolog implementations (see references in (Bruynooghe 1991)). However, the inherent space and time costs, which must be paid even when no backtracking occurs, impeded its introduction in real implementations.

For a long time, look-ahead methods dominated in solving constraint satisfaction problems. However, already in (Rosiers and Bruynooghe 1987) we have shown empirical evidence that look-back methods can be useful, even that it can be interesting 
to combine both. Starting in the nineties there is a renewed interest in look-back methods, e.g., (Ginsberg 1993), and in combining look-back with look-ahead e.g., (Dechter and Frost 2002).

Look-back turned out to be the most successful of the approaches tried in a research project aiming at detecting unsolvable queries (queries that do not terminate, such as the query $\leftarrow$ odd $(X)$, even $(X)$ for a program defining odd and even numbers). The approach was to construct a model of the program over a finite domain in which the query was false. The central part of this model construction was to search for a pre-interpretation leading to the desired model, i.e., with $D$ the domain, to find an appropriate function $D^{n} \rightarrow D$ for every n-ary functor in the program. A meta-interpreter was built which performed a backtracking search over the solution space. A control strategy was devised which resulted in the early detection of instances of program clauses which showed that the choices made so far could not result in the desired model. This meta-interpreter outperformed dedicated model generators on several problems (Bruynooghe et al. 1998). However it remained very sensitive to the initial ordering in which the various components of the different functions were assigned. The point was that not all choices made so far necessarily contributed to the evaluation of a clause instance. We experimented with constraint techniques and also investigated the use of intelligent backtracking. With a small programming effort, we could enhance the meta-interpreter to support a form of intelligent backtracking. As reported in (Bruynooghe et al. 1999), this was the most successful approach. As Prolog is a popular tool for prototyping search problems and as look-back methods, though useful, are not available in off-the-shelf Prolog systems, we decided to describe for a wider audience how to enhance a Prolog search program with a form of intelligent backtracking. The technique crucially depends on the impure feature of Prolog (assert/retract) that allows storing information when a dead end is reached. The stored information is used to decide whether a choice point should be skipped when chronological backtracking returns to it. Hence we propose the technique as a black pearl.

In the application mentioned above, the meta-interpreter is performing a substantial amount of computation after making a choice whereas the amount of computation added to support intelligent backtracking is comparatively small. This is not always the case. When the amount of computation in between choices is small and solutions are rather easy to find, the overhead of supporting intelligent backtracking may be larger than the savings due to the pruning of the search space. This is the case in toy problems such as the n-queens. In the example we develop here, there is a small speed-up.

We recall some basics of intelligent backtracking in Section 2. In Section 3, we introduce the example program and in Section 4 we enhance it with intelligent backtracking. We conclude with a discussion in Section 5 .

\section{Intelligent Backtracking}

Intelligent backtracking as described in (Bruynooghe 1981) is a very general schema. It keeps track of the reason for eliminating a variable in a domain. Upon reaching 
a dead end, it identifies a culprit for the failure and jumps back to the choice point where the culprit was assigned a value. Information about the variables assigned in between the culprit and the dead end can be retained if still valid, as in the dynamic backtracking of (Ginsberg 1993) which can be considered as an instance of the schema. More straightforward in a Prolog implementation is to give up that information, this gives the backjumping algorithm (Algorithm 3.3) in (Ginsberg 1993) (intelligent backtracking with static order in (Rosiers and Bruynooghe 1987)). We follow rather closely (Ginsberg 1993) for introducing it.

A constraint satisfaction problem (CSP) can be identified by a triple $(I, D, C)$ with $I$ a set of variables, $D$ a mapping from variables to domains and $C$ a set of constraints. Each variable $i \in I$ is mapped by $D$ into a domain $D_{i}$ of possible values. Each constraint $c \in C$ defines a relation $R_{c}$ over a set $I_{c} \subseteq I$ of variables and is satisfied for the tuples in that relation. A solution to a CSP consists of a value $v_{i}$ (an assignment) for each variable $i$ in $I$ such that: (1) for all variables $i: v_{i} \in D_{i}$ and, (2) for all constraints $c$ : with $I_{c}=\left\{j_{1}, \ldots, j_{k}\right\}$, it holds that $\left(v_{j_{1}}, \ldots, v_{j_{k}}\right) \in R_{c}$.

A partial solution to a $\operatorname{CSP}(I, D, C)$ is a subset $J \subseteq I$ and an assignment to each variable in $J$. A partial solution $P$ is ordered by the order in which the algorithm that computes it assigns values to the variables and is denoted by a sequence of ordered pairs $\left(i, v_{i}\right)$. A pair $\left(i, v_{i}\right)$ indicates that variable $i$ is assigned value $v_{i}$; $I_{P}=\left\{i \mid\left(i, v_{i}\right) \in P\right\}$ denotes the set of variables assigned values by $P$.

Given a partial solution $P$, an eliminating explanation (cause-list in (Bruynooghe 1981)) for a variable $i$ is a pair $\left(v_{i}, S\right)$ where $v_{i} \in D_{i}$ and $S \subseteq I_{P}$. It expresses that the assignments to the variables of $S$ by the partial solution $P$ cannot be extended into a solution where variable $i$ is assigned value $v_{i}$. Contrary to (Ginsberg 1993), we use an elimination mechanism that tests one value at a time. Hence we assume a function consistent $\left(P, i, v_{i}\right)$ that returns true when $P \cup\left\{\left(i, v_{i}\right)\right\}$ satisfies all constraints over $\left.I_{P} \cup\{i\}\right)$ and a function $\operatorname{elim}\left(P, i, v_{i}\right)$ that returns an eliminating explanation $\left(v_{i}, S\right)$ when $\neg$ consistent $\left(P, i, v_{i}\right)$.

Below, we formulate the backjumping algorithm; next we clarify its reasoning. $E_{i}$ is the set of eliminating explanations for variable $i$.

\section{Algorithm 1}

Given as inputs a CSP $(I, D, C)$.

1. Set $P:=\emptyset$.

2. If $I_{P}=I$ return $P$. Otherwise select a variable $i \in I \backslash I_{P}$, set $S_{i}:=D_{i}$ and $E_{i}:=\emptyset$.

3. If $S_{i}$ is empty then go to step 4 ; otherwise, remove an element $v_{i}$ from it. If consistent $\left(P, i, v_{i}\right)$ then extend $P$ with $\left(i, v_{i}\right)$ and go to step 2; otherwise add $\operatorname{elim}\left(P, i, v_{i}\right)$ to $E_{i}$ and go to step 3.

4. ( $S_{i}$ is empty and $E_{i}$ has an eliminating explanation for each value in $D_{i}$.) Let $C$ be the set of all variables appearing in the explanations of $E_{i}$.

5. If $C=\emptyset$, return failure. Otherwise, let $\left(l, v_{l}\right)$ be the last pair in $P$ such that $l \in C$. Remove from $P$ this pair and any pair following it. Add $\left(v_{l}, C \backslash\{l\}\right)$ to $E_{l}$, set $i:=l$ and go to step 3 . 
In step 3, when the extension of the partial solution is inconsistent then $\operatorname{elim}\left(P, i, v_{i}\right)$ returns a pair $\left(v_{i},\left\{j_{1}, \ldots, j_{m}\right\}\right)$ such that the partial solution $\left(j_{1}, v_{j_{1}}\right), \ldots,\left(j_{m}, v_{j_{m}}\right),\left(i, v_{i}\right)$ violates the constraints. The inconsistency of this assignment can be expressed by the clause: $\leftarrow j_{1}=v_{j_{1}}, \ldots, j_{m}=v_{j_{m}}, i=v_{i}$ (The head is false, the body is a conjunction).

In step 4 , when $S_{i}$ is empty, we have an eliminating explanation for each value $v_{i_{k}}$ in the domain $D_{i}$. Hence we have a set of clauses of the form

$$
\leftarrow j_{k, 1}=v_{j_{k, 1}}, \ldots, j_{k, m_{k}}=v_{j_{k, m_{k}}}, i=v_{i_{k}}
$$

The condition that the variable $i$ must be assigned a value from domain $D_{i}$ with $n$ elements can be expressed by the clause (the head is a disjunction, the body is true):

$$
i=v_{i_{1}}, \ldots, i=v_{i_{n}} \leftarrow
$$

Now, one can perform hyperresolution (Robinson 1965) between clause (2) and the clauses of the form (1) (for $k$ from 1 to $n$ ). This gives:

$$
\leftarrow j_{1,1}=v_{j_{1,1}}, \ldots, j_{1, m_{1}}=v_{j_{1, m_{1}}}, \ldots, j_{n, 1}=v_{j_{n, 1}}, \ldots, j_{n, m_{n}}=v_{j_{n, m_{n}}}
$$

This expresses a conflict between the current values of the variables in the set $\left\{j_{1,1}, \ldots, j_{1, m_{1}}, \ldots, j_{n, 1}, \ldots, j_{n, m_{n}}\right\}=C$. Hence, with $l$ the last assigned variable in $C, C \backslash\{l\}$ is an eliminating explanation for $v_{l}$. The conflict $C$ is computed in step 4. When empty, the problem has no solution as detected in step 5. Otherwise, step 5 backtracks and adds the eliminating explanation $\left(v_{l}, C \backslash\{l\}\right)$ to the set of eliminating explanations of variable $l$.

One can observe that the algorithm does not use the individual eliminating explanations in the set $E_{i}=\left(v_{i_{k}}, S_{k}\right)$, but only the set $C$ which is the union of the sets $S_{k}$. As we have no interest in introducing more refined forms of intelligent backtracking, we develop Algorithm 2 where $E_{i}$ holds the union of the sets $S_{k}$ in the eliminating explanations of variable $i$. To obtain an algorithm that closely corresponds to the Prolog encoding we present in Section 4, we reorganise the code and introduce some more changes. The function $\operatorname{elim}\left(P, i, v_{i}\right)$ that returns an eliminating explanation $\left(v_{i}, S\right)$ for the current value of variable $i$ is replaced by a function $\operatorname{conflict}\left(P, i, v_{i}\right)$ that returns the set $\{i\} \cup S$ (the variables that participate in a conflict as represented by Equation 1). This conflict is stored in a variable $C$ (step 3 of Algorithm 2). It is nonempty and $i$ is the last assigned variable, hence the value of $i$ remains unchanged in step 4 and, in step 5, the eliminating explanation $C \backslash\{i\}$ is added to $E_{i}$. This reorganisation of the code has as result that a local conflict (the chosen value for the last assigned variable $i$ is inconsistent with the partial solution) and a deep conflict (all values for variable $i$ have been eliminated) are handled in a uniform manner: upon failure, the algorithm computes a conflict and stores it in variable $C$ (for the local conflict in step 3, for the deep conflict in step 5), backtracks to the variable computed in step 4 (the "culprit") and resumes in step 5 with updating $E_{i}$ and trying a next assignment to variable $i$. 
Algorithm 2

Given as input a $\operatorname{CSP}(I, D, C)$.

1. Set $P:=\emptyset$.

2. If $I_{P}=I$ return P. Otherwise select a variable $i \in I \backslash I_{P}$. Select a value $v_{i}$ from $D_{i}$. Set $S_{i}:=D_{i} \backslash\left\{v_{i}\right\}$ and $E_{i}:=\emptyset$.

3. If consistent $\left(P, i, v_{i}\right)$ then extend $P$ with $\left(i, v_{i}\right)$ and go to step 2; otherwise set $C:=\operatorname{conflict}\left(P, i, v_{i}\right)$.

4. If $C=\emptyset$ then return failure; otherwise let $\left(l, v_{l}\right)$ be the last pair in $P$ such that $l \in C$. Set $i:=l$.

5. Add $C \backslash\{i\}$ to $E_{i}$. If $S_{i}=\emptyset$ then $C:=E_{i}$ and go to step 4; otherwise select and remove a value $v_{i}$ from $S_{i}$ and go to step 3 .

\section{A search problem}

The code below is, apart from the specific constraints, fairly representative for a finite domain constraint satisfaction problem. The problem is parameterized with two cardinalities: VarCard, the number of variables (the first argument of problem/3) and ValueCard, the number of values in the domains of the variables (the second argument of problem/3). The third argument of problem/3 gives the solution in the form of a list of elements assign $\left(i, v_{i}\right)$. The main predicate uses init_domain/2 to create a domain $[1,2, \ldots$, ValueCard $]$ and init_pairs/3 to initialize Pairlist as a list of pairs $i-D_{i}$ with $D_{i}$ the domain of variable $i$. The first argument of extend_solution/3 is a list of pairs $i-D_{i}$ with $i$ an unassigned variable and $D_{i}$ what remains of its domain; the second argument is the (consistent) partial solution (initialized as the empty list) and the third argument is the solution. The predicate is recursive; each iteration extends the partial solution with an assignment to the first variable on the list of variables to be assigned. The nondeterministic predicate my_assign/2 selects the value. If desirable, one could introduce a selection function which dynamically selects the variable to be assigned next.

Consistency of the new assignment with the partial solution is tested by the predicates consistent $1 / 2$ and consistent $2 / 2$. They create a number of binary constraints. The binary constraints themselves are tested with the predicates constraint $1 / 2$ and constraint $2 / 2$. What they express is not so important. The purpose is to create a problem that is sufficiently difficult so that enhancing the program with intelligent backtracking pays off. For the interested reader, the predicate consistent $2 / 2$ creates a very simple constraint that verifies (using constraint1/2) that the value of the newly assigned variable is different from the value of the previously assigned variable. The predicate consistent $1 / 2$ creates a set of more involved constraints. The odd numbered and even numbered variables each encode the constraints of the n-queens problem. As a result, the solution of e.g., problem $(16,8, \mathrm{~S})$ contains a solution for the 8-queens problem in the odd numbered variables and a different (due to the constraints created by consistent2/2) solution in the even numbered variables. Substantial search is required to find a first solution. For example, the first solution for problem $(16,8, S)$ 
is found after 32936 assignments (using a similar set-up of constraints, a solution is found for the 8-queen problem after only 876 assignments).

Note that the constraint checking between the new assigned variable and the other assigned variables is done in an order that is in accordance with the order of assigning variables. Hence consistent1/2 is not tail recursive. The order is not important for the algorithm without intelligent backtracking. However, it is crucial to obtain optimal intelligent backtracking: as with chronological backtracking, constraint checking will stop at the first conflict detected and an eliminating explanation will be derived from it. As an eliminating explanation with an older assigned variable gives more pruning than one with a more recently assigned variable, the creation of constraints requires one to pay attention to the order. It is done already here to minimize the differences between this version and the enhanced version.

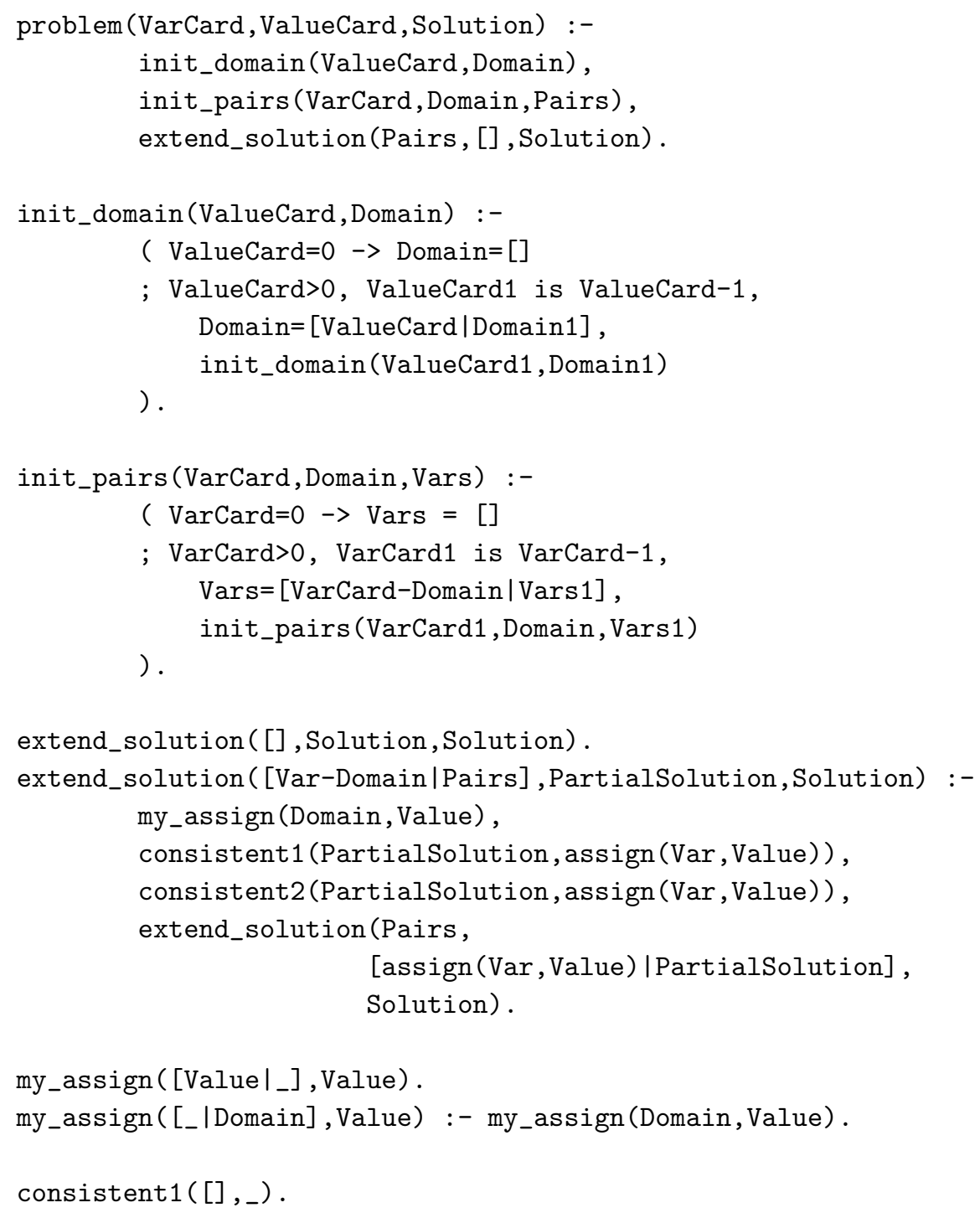




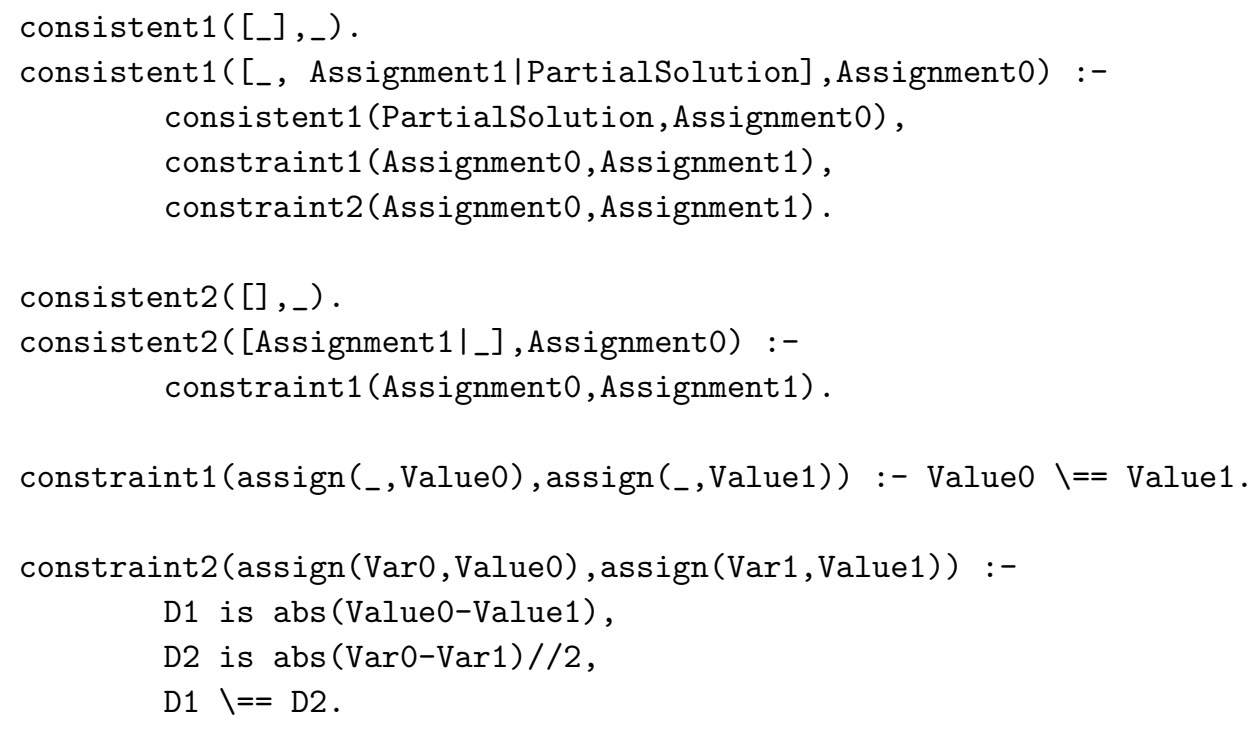

\section{Adding intelligent backtracking}

Adding intelligent backtracking requires us to maintain eliminating explanations. In Algorithm 2, a single eliminating explanation is associated with each variable. The eliminating explanation of a variable $i$ is initialised as empty in step 2, when assigning a first value to the variable. It is updated in step 5, when the last assigned value turns out to be the "culprit" of an inconsistency. This happens just before assigning the next value to variable $i$. This indicates that the right place to store eliminating explanations is as an extra argument in the predicate my_assign/2. In step 4, the algorithm has to identify the "last" variable $l$ of a conflict (the "culprit"), just before updating the eliminating explanation. We will also use the my_assign/2 predicate to check whether the variable it assigns corresponds to the culprit of the failure. Hence also the identitity of the variable should be an argument. These considerations lead to the replacement of the my_assign/2 predicate by the following my_assign/4 predicate.

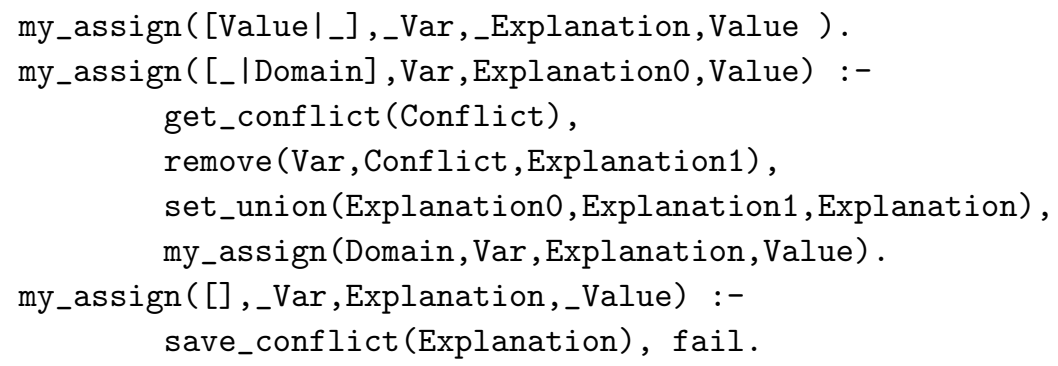

It is called from extend_solution/4 as myassign(Domain, Var, [] , Value) (what remains of the domain is the first argument, the second argument is the variable being assigned, the third argument is the initially empty eliminating explanation and the fourth argument returns the assigned value). The initial call together with 
the base case perform the otherwise branch of step 2. The second clause, entered upon backtracking when the domain is nonempty, checks whether the variable being assigned is the culprit. To do so, it needs the conflict. As this information is computed just before failure occurs, it cannot survive backtracking when using the pure features of Prolog. One has to rely on the impure features for asserting/updating clauses. Either assert/1 and retract/1 or more efficient variants of specific Prolog systems ${ }^{1}$. The call to get_conflict(Conflict) picks up the saved conflict ${ }^{2}$; next, the call remove (Var, Conflict,Explanation1) checks whether Var is part of it. If not, my_assign/4 fails and backtracking returns to the previous assignment. If Var is the culprit, then the code performs step 5 of the algorithm: remove/3 returns the eliminating explanation in its third argument, set_union/3 adds it to the current eliminating explanation and the recursive call checks whether the domain is empty. If not, the base case of my_assign/4 assigns a new value. If the domain is empty, then the last clause is selected. The eliminating explanation becomes the conflict and is saved with the call to save_conflict(Explanation) that relies on the impure features ${ }^{3}$ and the clause fails.

Further modifications are in the predicates constraint1/2 and constraint2/2 that perform the constraint checking. If a constraint fails, the variables involved in it make up the conflict and have to be saved so that after re-entering myassign/4 the conflict can be picked up and used to compute an eliminating explanation (step $3)$. As the last assigned variable participates in all constraints, it is part of the conflict. For example, the code for constraint1/2 becomes:

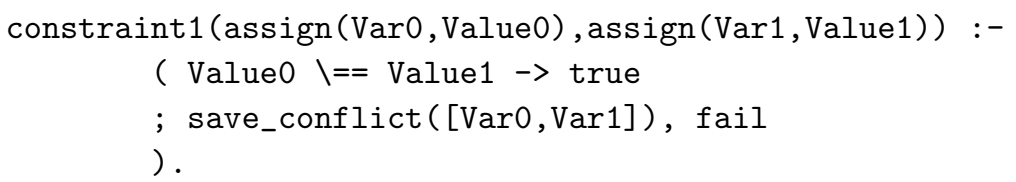

The modification to constraint2/2 is similar. Recall that the order in which constraints are checked determines the amount of pruning that is achieved. Finally, if one is interested in more than one solution then also a conflict has to be stored when finding a solution. It consists of all variables making up the solution. Using a predicate allvars/2 that extracts the variables from a solution, the desired behavior is obtained as follows:

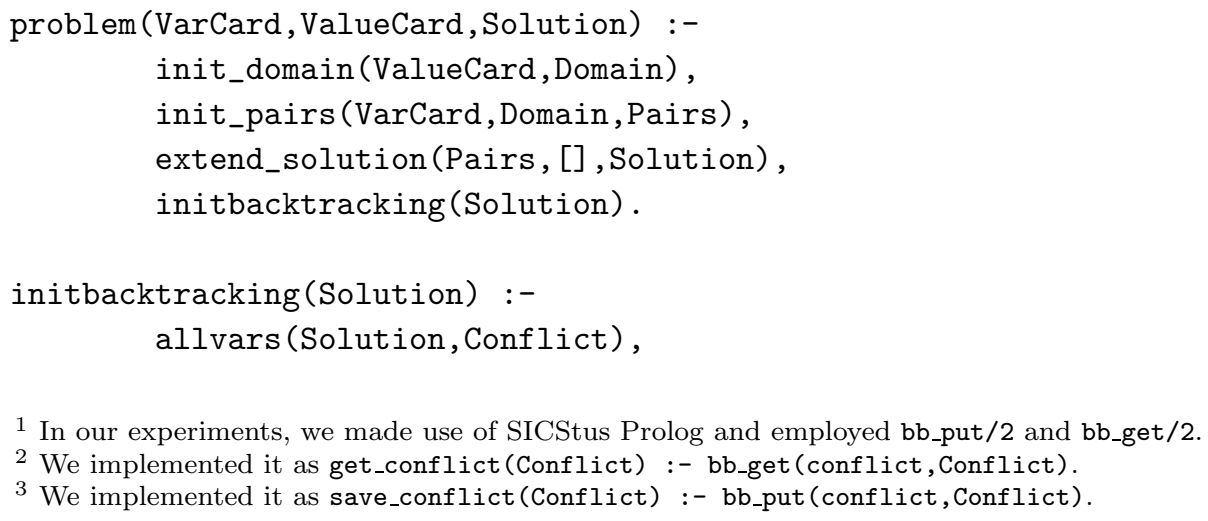


save_conflict(Conflict).

The enhanced program generates the same solutions as the original, and in the same order. For problem $(16,8, \mathrm{~S})$ the number of assignments goes down from 32936 to 4015 and the execution time from $140 \mathrm{~ms}$ to $70 \mathrm{~ms}$; for problem $(20,10, \mathrm{~S})$, the reduction is respectively from 75950 to 15813 and from $370 \mathrm{~ms}$ to $310 \mathrm{~ms}$. The achieved pruning more than compensates for the (substantial) overhead of recording and updating conflicts ${ }^{4}$ and of the calls to remove/3 and set_union/3. Note that the speed-up decreases with larger instances of this problem. This is likely due to the increasing overhead of the latter two predicates. Keeping the conflict set sorted (easy here because the variable numbers corresponds with the order of assignment) such that the culprit is always the first element could reduce that overhead.

\section{Discussion}

In this black pearl, we have illustrated by a simple example how a chronological backtracking algorithm can be enhanced to perform intelligent backtracking. As argued in the introduction, look-back techniques are useful in solving various search problems. Hence exploring their application can be very worthwhile when building a prototype solution for a problem. The technique presented here illustrates how this can be realized with a small effort when implementing a prototype in Prolog. Interestingly, the crucial feature is the impurity of Prolog that allows the search to transfer information from one point in the search tree (a dead end) to another. It illustrates that Prolog is a multi-faceted language. On the one hand it allows for pure logic programming, on the other hand it is a very flexible tool for rapid prototyping. Note that the savings due to the reduction of the search space could be undone by the overhead of computing and maintaining the extra information, especially, when the amount of computation between two choice points is small.

The combination of look-back and look-ahead techniques can be useful, and algorithms integrating both can be found, e.g., (Dechter and Frost 2002). The question arises whether our solution can be extended to incorporate look-ahead. This requires some work, however, much of the design can be preserved. The initialization (init_domains/3) should not only associate variables with their initial finite domain, but also with their eliminating explanations (initially empty). Then the code for the main iteration could be as follows:

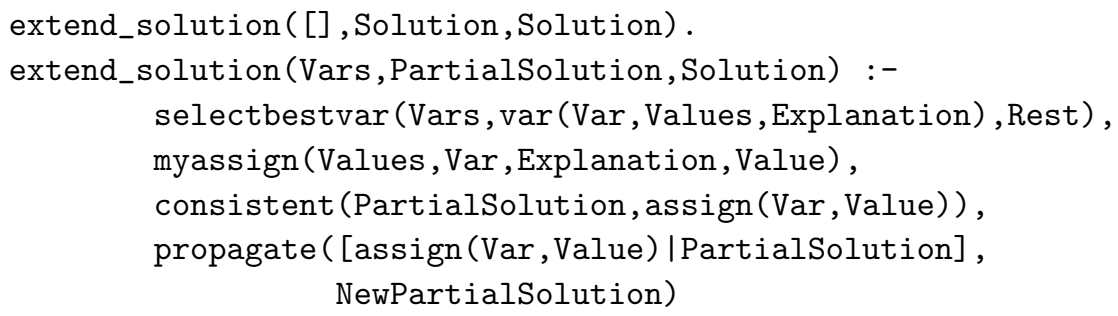

\footnotetext{
4 Using bb_get and bb_put to count the number of assignments increases execution time of the initial algorithm for problem $(16,8, \mathrm{~S})$ from $140 \mathrm{~ms}$ to $400 \mathrm{~ms}$.
} 
extend_solution(Vars, NewPartialSolution, Solution) .

The predicate selectbestvar/3 is used to dynamically select the next variable to assign. It returns the identity of the variable $(\mathrm{Var})$, the available values (Values) and the explanation (Explanation) for the eliminated values. When a partial solution is successfully extended, the predicate propagate/2 has to take care of the constraint propagation: eliminating values from domains and updating the corresponding explanations after which the next iteration can start. Computing the eliminating explanation for each eliminated value requires great care and depends on the kind of look-ahead technique used. It is pretty straightforward for forward checking but requires careful analysis in case of e.g., arc consistency as no pruning will occur on backjumping when the elimination is attributed to all already assigned variables.

\section{Acknowledgments}

I am grateful to Bart Demoen, Gerda Janssens and Henk Vandecasteele for useful comments on various drafts of this pearl. I am very grateful to the reviewers. Indeed, as often is the case, their persistence and good advise greatly contributed to the clarity of the exposition.

\section{References}

Bruynooghe, M. 1981. Solving combinatorial search problems by intelligent backtracking. Information Processing Letters 12, 1, 36-39.

Bruynooghe, M. 1991. Intelligent backtracking revisited. In Computational Logic, Essays in Honor of Alan Robinson, J.-L. Lassez and G. Plotkin, Eds. MIT Press, 166177.

Bruynooghe, M. And Pereira, L.-M. 1984. Deduction revision by intelligent backtracking. In Implementation of Prolog, J. Campbell, Ed. Ellis Horwood, 194-215.

Bruynooghe, M., Vandecasteele, H., de WaAl, D. A., And Denecker, M. 1998. Detecting unsolvable queries for definite logic programs. In Principles of Declarative Programming, Proc. PLILP'98 and ALP'98, C. Palamidessi, H. Glaser, and K. Meinke, Eds. LNCS. Springer, 118-133.

Bruynooghe, M., Vandecasteele, H., de WaAl, D. A., And Denecker, M. 1999 Detecting unsolvable queries for definite logic programs. J. Functional and Logic Programming 1999, 1-35.

Dechter, R. And Frost, D. 2002. Backjump-based backtracking for constraint satisfaction problems. Artificial Intelligence 136, 2, 147-188.

Ginsberg, M. L. 1993. Dynamic backtracking. Journal of Artificial Intelligence Research 1, 25-46.

Robinson, J. A. 1965. Automated deduction with hyper-resolution. Int. J. Computh. Math 1, 227-234.

Rosiers, W. And Bruynooghe, M. 1987. Empirical study of some constraint satisfaction algorithms. In Artificial Intelligence II, Methodology, Systems, Applications, Proc. AIMSA'86, P. Jorrand and V. Sgurev, Eds. North Holland, 173-180.

Van Hentenryck, P. 1989. Constraint Satisfaction in Logic Programming. MIT Press. 